\begin{tabular}{|c|l|}
\hline Title & A pplication of the nitroanisole as an infrared detector used in middle infrared interferometer \\
\hline Author(s) & Miyamoto, Naoki; Nisiyama, Shusuke; Tomioka, Satoshi; Enoto, Takeaki \\
\hline Citation & $\begin{array}{l}\text { Optics Communications, 260(1), 25-29 } \\
\text { https://doi.org/40.1016j.optcom.2005.10.031 }\end{array}$ \\
\hline Issue Date & 2006-04_01 \\
\hline Doc URL & http://hdl.handle.net/2115/8493 \\
\hline Type & article(author version) \\
\hline File Information & OptCom260_p25.pdf \\
\hline
\end{tabular}

Instructions for use 


\title{
Application of the nitroanisole as an infrared detector used in middle infrared interferometer.
}

\author{
Naoki Miyamoto*, Shusuke NISIYAMA, Satoshi TOMIOKA, \\ Takeaki ENOTO \\ Graduate School of Engineering, Hokkaido University, Sapporo 060-8628, Japan
}

\begin{abstract}
We propose the application of nitroanisole as a detector for middle infrared (midIR) interferometry or holography. The present experiment utilizes the liquid form of nitroanisole, which has a thermal lens effect, i.e. a temperature dependent refractive index. Since the nitroanisole absorbs IR radiation as heat, it is possible to estimate the IR intensity distribution on the nitroanisole from the diffraction pattern made by visible laser light that is transmitted through the nitroanisole. In this study, the time resolution and the diffraction efficiency of the nitroanisole was measured under various conditions. The experimental results show that the nitroanisole has a time resolution as high as that of a standard video camera, as well as a high diffraction efficiency and the spatial resolution equivalent to that of a conventional IR camera. Furthermore, we confirmed that the phase shift in mid-IR region can be estimated by analyzing the change in the visible diffraction pattern.
\end{abstract}

Key words: Nitroanisole; Interferometer; Infrared detectors

\section{Introduction}

In recent years, many devices that can detect infrared (IR) radiation have been invented. Detectors that contain such devices as pyroelectric devices and $\mathrm{HgCdTe}$ diodes have been used in applications of IR laser interferometry[1-3], non-destructive inspection[4], medical diagnosis[5], etc. In particular, interferometry is very effective for phase measurement, and some studies on plasma

\footnotetext{
* Corresponding author. Fax: +81-11-706-7128

Email address: nao@athena.qe.eng.hokudai.ac.jp (Naoki Miyamoto).
}

Preprint submitted to Elsevier Science 14 October 2005 
measurement[1,2] or digital holography[6] using a $\mathrm{CO}_{2}$ laser of wavelength $10.6 \mu \mathrm{m}$ have been reported. However, some of the devices used in these techniques have various restrictions, for instance, that they must be used in a cryogenic system, or that the detectors must be arranged in a matrix in order to obtain a two-dimensional image in a single trial. We propose the use of nitroanisole as a much simpler device with purpose of detecting mid-IR radiation. The nitroanisole exhibits a thermal lens effect[7-9], in which the refractive index is dependent upon temperature. This effect results in phase modulation on visible light, in direct response to intensity of the incident IR radiation, which is absorbed as heat. In the case of IR laser interferometry, interference fringe patterns that are created by the IR laser on the nitroanisole are observed as the refractive index distribution, hence the nitroanisole functions as a phase grating for visible light. It is, therefore, conceivable to estimate the IR intensity distribution on the nitroanisole by analyzing the diffraction pattern made by a visible laser, which is transmitted through the nitroanisole. Also, since a two-dimensional device using the nitroanisole does not require matrix structure, it is expected that the measurement system must have high spatial resolution, equivalent to that of existing IR cameras.

The purpose of this paper is to investigate the time resolution and the diffraction efficiency of the nitroanisole for a detector in mid-IR spectrum range. In addition, we attempt to estimate the phase shift in mid-IR region based on the change of visible diffraction patterns.

\section{Characteristics of the nitroanisole}

The nitroanisole has three positions of $o^{-}, m^{-}$, and $p$-nitroanisole. We propose to use $o$-nitroanisole which is a colorless or slightly yellow liquid with a boiling point of $277^{\circ} \mathrm{C}$, a melting point of $9.4^{\circ} \mathrm{C}$, and molecular weight of 153.14 , as a mid-IR detector. The $o$-nitroanisole in our experiment has significant thermal lens effect, which means the refractive index for visible light is dependent on temperature. We tested that the refractive index of $o$-nitroanisole varied according to temperature at a rate of $-1.1 \times 10^{-3}[1 / \mathrm{K}]$ as shown in Fig.1. In addition we confirmed that it has an absorption band in mid-IR region by infrared absorption spectroscopy and that it hardly absorbs visible light. Therefore when the nitroanisole is irradiated with the IR radiation, its temperature rises, causing its refractive index to decreases. 


\section{Experiments and results}

\section{1 time resolution}

The experimental setup for measuring the time resolution and the diffraction efficiency is shown in Fig.2. The nitroanisole was sandwiched between the glass substrate and ZnSe, an IR transmitting material. The thickness between the two substrates was adjusted by a film spacer and determined to be about $100 \mu \mathrm{m}$. Linearly polarized light from a c.w.(continuous wave) $\mathrm{CO}_{2}$ laser of $10.6 \mu \mathrm{m}$ wavelength was divided into two beams of roughly equal intensity by a beam splitter and the two beams were superimposed onto the nitroanisole cell at the appropriate angle to create the interference fringe pattern on the nitroanisole, which was illuminated by a He-Ne laser of $632.8 \mathrm{~nm}$ wavelength. Accordingly, since a refractive index distribution with periodic variation according to the interference fringe pattern was constructed on the nitroanisole, we were able to observe the diffracted He-Ne laser light. We measured the time variation of the intensity of the first-order diffraction He-Ne laser which was induced by the interference of the $\mathrm{CO}_{2}$ laser with a photo detector. A mechanical shutter unit was located outside of the $\mathrm{CO}_{2}$ laser resonator in order to avoid any possible sudden change to the $\mathrm{CO}_{2}$ laser power caused by Q-switched oscillation. One graph of the measured time variation of diffracted He-Ne light intensity is shown in Fig.3. Irradiation time of the $\mathrm{CO}_{2}$ laser was about $500 \mathrm{~ms}$. The diffraction efficiency, the rise time, and the fall time were defined respectively as the intensity ratio of the first-order diffraction light to that of zero-order, the time to rise from the initial state to $90 \%$ of static level, and the time of decrease to $10 \%$ of static level. In order to understand the dependencies of the diffraction efficiencies on both spatial frequency of interference fringe and laser power density, both the angle between two $\mathrm{CO}_{2}$ laser beams and the laser output power density were changed.

Fig. 4 shows the dependency of the $\mathrm{CO}_{2}$ laser output power density on the time resolution of the nitroanisole. The spatial frequency of the interference fringe made by the $\mathrm{CO}_{2}$ laser on the nitroanisole was $2 \mathrm{lp} / \mathrm{mm}$. This result indicates that the rise time becomes shorter and the fall time becomes longer in response to an increase in the $\mathrm{CO}_{2}$ laser output power density. When the $\mathrm{CO}_{2}$ laser output power density was increased from $0.5 \mathrm{~W} / \mathrm{cm}^{2}$ to $3.0 \mathrm{~W} / \mathrm{cm}^{2}$, the rise time changed from $45 \mathrm{~ms}$ to $36 \mathrm{~ms}$ and the fall time changed from $14 \mathrm{~ms}$ to $24 \mathrm{~ms}$. We surmised that the reason why the rise time became shorter is that the time to reach thermal equilibrium state is reduced with higher output power density. On the other hand, the reason why the fall time became longer is that more time to return to homogeneous temperature distribution was needed since the total irradiated power was larger. This reasoning is based on the assumption that the difference of the temperature induced by the interference of the $\mathrm{CO}_{2}$ 
laser in the nitroanisole is greater in case of high laser power density.

The dependency of the time resolution on the spatial frequency is shown in Fig.5. The power density of the $\mathrm{CO}_{2}$ laser used was $2.0 \mathrm{~W} / \mathrm{cm}^{2}$ for all measurements. Both the rise time and the fall time became shorter as the spatial frequency of the interference fringes on the nitroanisole increased. When the spatial frequency of the interference fringes was increased from $0.5 \mathrm{lp} / \mathrm{mm}$ to $5.5 \mathrm{lp} / \mathrm{mm}$, the rise time changed from $40 \mathrm{~ms}$ to $12 \mathrm{~ms}$ and the fall time changed from $20 \mathrm{~ms}$ to $8 \mathrm{~ms}$. Both the rise time and the fall time became shorter in response to an increase in spatial frequency, because the relative temperature difference within the nitroanisole at the thermal equilibrium state is small in case of high spatial resolution. Therefore, it is understood that the time required to reach to the thermal equilibrium state and the time required to return to the homogeneous temperature distribution were both significantly reduced.

From above results, we confirmed that the nitroanisole has a high time resolution, roughly equivalent to that of a video camera in some conditions. It is expected that the nitroanisole can detect a phase shift of the mid-IR region in real time.

\section{2 diffraction efficiency}

Fig.6 shows the experimental result of diffraction efficiency measurements. In the case of high laser power density and low spatial frequency, a relatively high diffraction efficiency of about $17 \%$ was obtained, compared with the amplitude diffraction gratings. On the other hand, in case of a high spatial frequency of more than $8 \mathrm{lp} / \mathrm{mm}$, the diffraction efficiency was less than $1 \%$. Since the temperature difference in the thermal equilibrium state was small in high spatial resolution, the phase modulation to the He-Ne laser was small.

It was observed that the nitroanisole has high spatial resolution of at least $8 \mathrm{lp} / \mathrm{mm}$, which is higher than that of conventional IR cameras based on pyroelectric devices, generally about $5 \mathrm{lp} / \mathrm{mm}$. When we consider the technical requirement for IR holography, a detector capable of spatial resolution on the order of the wavelength of the light source is necessary. In this experiment, we were not able to demonstrate that the nitroanisole has sufficiently high spatial resolution to satisfy such a requirement. However, it can be used as the detector in an in-line type interferometer such as a Mach-Zehnder system, because the detector in this type of interferometer that has a spatial resolution capability of about 10 times that of the wavelength is sufficient to detect the fringe shift. 
Our experiments confirmed that the nitroanisole performed as a usual detector in the case of IR interferometry for the purpose of measuring phase shift in the IR region. The experimental setup for measuring phase shift in the IR region is shown in Fig.7. The setup consists of a Mach-Zehnder interferometer in which the light source is a c.w. $\mathrm{CO}_{2}$ laser. The He-Ne laser magnified by a lens with focal length of $3 \mathrm{~cm}$ was projected through the nitroanisole cell in which an interference fringe of about $2 \mathrm{lp} / \mathrm{mm}$ had been made with the $\mathrm{CO}_{2}$ laser. The power density of the $\mathrm{CO}_{2}$ laser was $2.5 \mathrm{~W} / \mathrm{cm}^{2}$. The distance from the lens to the cell was $10 \mathrm{~cm}$ and that from the cell to the screen was $90 \mathrm{~cm}$. The image in Fig.7 is the diffraction pattern observed on the screen.

First, for comparison with experimental result, we calculated the theoretical diffraction pattern from Kirchhoff's diffraction theory, namely

$$
u(x, y)=A \iint_{\Gamma} g(\xi, \eta) \frac{\exp (i k s)}{s}(\cos (n s)-\cos (n r)) d \xi d \eta
$$

where $A$ is a complex constant, $s$ is the distance from the focal point of the lens to one point $(\xi, \eta)$ on the nitroanisole, $r$ is a distance from the point $(\xi, \eta)$ to one point $(x, y)$ on the screen, $n$ is the orthogonal direction of the cell, $n s$ is the angle between $n$ and $s$, and $n r$ is the angle between $n$ and $r$, as shown in Fig.7. In Eq. $(1), g(\xi, \eta)$ is a wavefront of the He-Ne laser through the nitroanisole given by,

$$
g(\xi, \eta)=\frac{\exp (i k r)}{r} \exp \{i \alpha I(\xi, \eta)\}
$$

where $I(\xi, \eta)$ is the IR intensity distribution on the nitroanisole, and $\alpha$ is a coefficient related to the phase modulation. We conducted a one-dimensional analysis of the diffraction pattern. In this analysis we assumed that the maximum phase shift imparted by the nitroanisole to the He-Ne laser beam is $0.6 \pi$, i.e., $\alpha=0.6 \pi / I(\xi, \eta)_{\max }$. The calculated pattern is shown in Fig.8. An experimental result is also shown in the figure. Both of these results were normalized with reference to their respective maximum intensity. The experimental diffraction pattern was recorded by a digital camera and we selected only the central portion in the fringe direction to obtain the distribution along the perpendicular axis to the fringe direction. In order to reduce noise, the intensity at each position along the perpendicular axis was taken as the average of 20 pixels along the fringe direction. The experimental and calculated results show good agreement in the center region of the pattern.

Next, we carried out an experiment for the purpose of estimating phase shift 
in the mid-IR region based on the change of diffraction pattern. We added a ZnSe plate in order to vary the optical path length of mid-IR light on the $\mathrm{CO}_{2}$ laser path in the Mach-Zehnder interferometer as shown in Fig.7. The thickness of the $\mathrm{ZnSe}$ plate was $3.2 \mathrm{~mm}$. In response to the rotation of the ZnSe plate, the interference fringe on the nitroanisole shifted. Accordingly, the diffraction pattern on the screen also shifted. We determined the best fitting periodic function to match the experimental, this function was used to represent the shift of the visible diffraction pattern corresponding to the phase shift of mid-IR radiation. The relationship between the phase shift of mid-IR radiation and the shift of the visible diffraction pattern is shown in Fig.9. This result shows that the phase shift of the visible diffraction pattern is identical to the phase shift of mid-IR light rays. Therefore, we conclude that it is possible to estimate the phase shift of the mid-IR region from the phase shift of the visible diffraction pattern.

\section{Conclusions}

We proposed a method to use nitroanisole as a detector for mid-IR interferometry or holography applications. Experimental results demonstrated that the nitroanisole performs well as a mid-IR detector, because the nitroanisole has high spatial resolution, time resolution and diffraction efficiency compared with conventional detectors. In addition, we demonstrated that it is not difficult to estimate the phase shift of the mid-IR region with the nitroanisole, and therefore, nitroanisole is considered feasible for applications in mid-IR interferometry. 


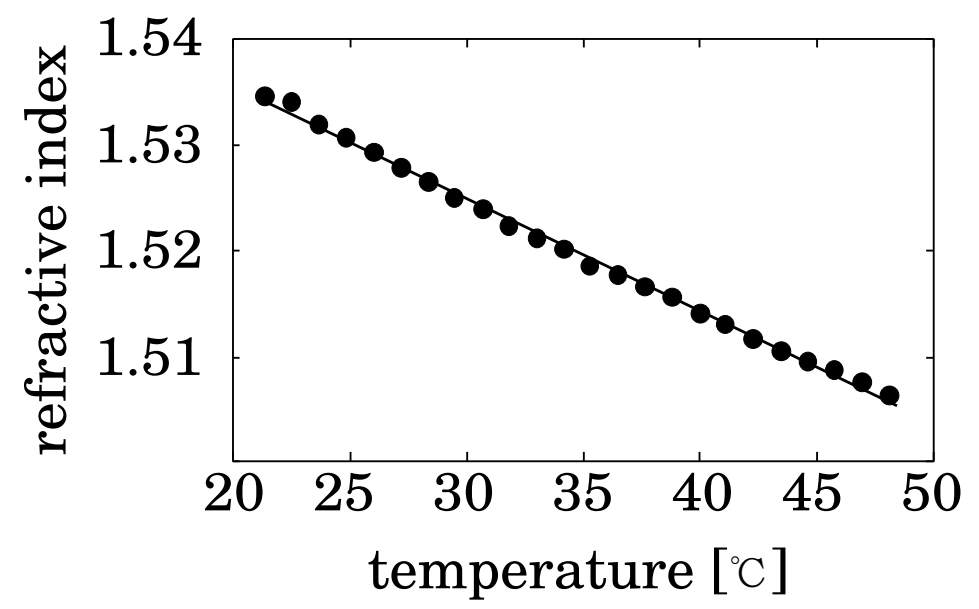

Fig. 1. Temperature dependence of the refractive index of the nitroanisole. 


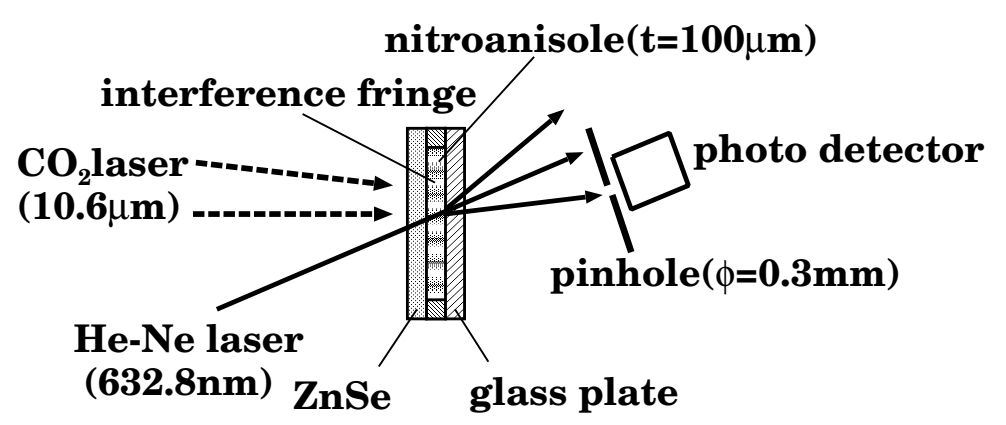

Fig. 2. Experimental setup for measuring the time resolution and the diffraction efficiency. 


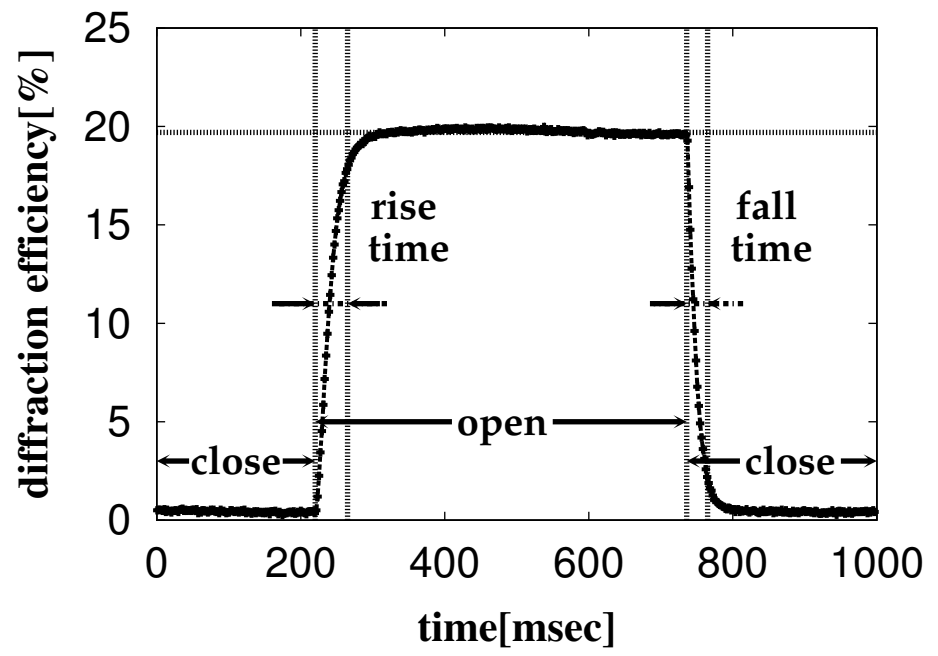

Fig. 3. Time variation of the first-order diffraction of He-Ne laser light intensity 


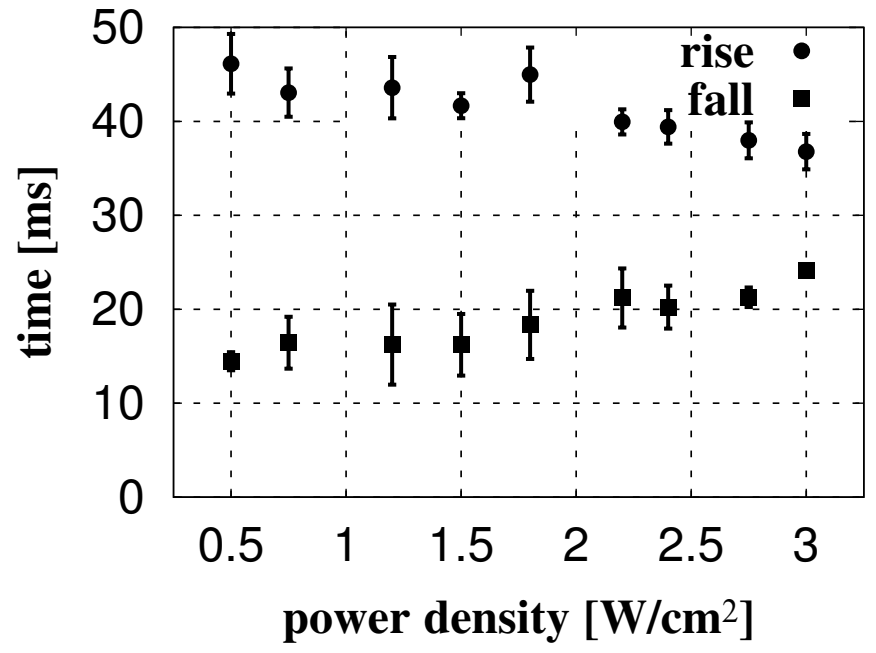

Fig. 4. The time resolution of the nitroanisole as a function of the $\mathrm{CO}_{2}$ laser output power density. 


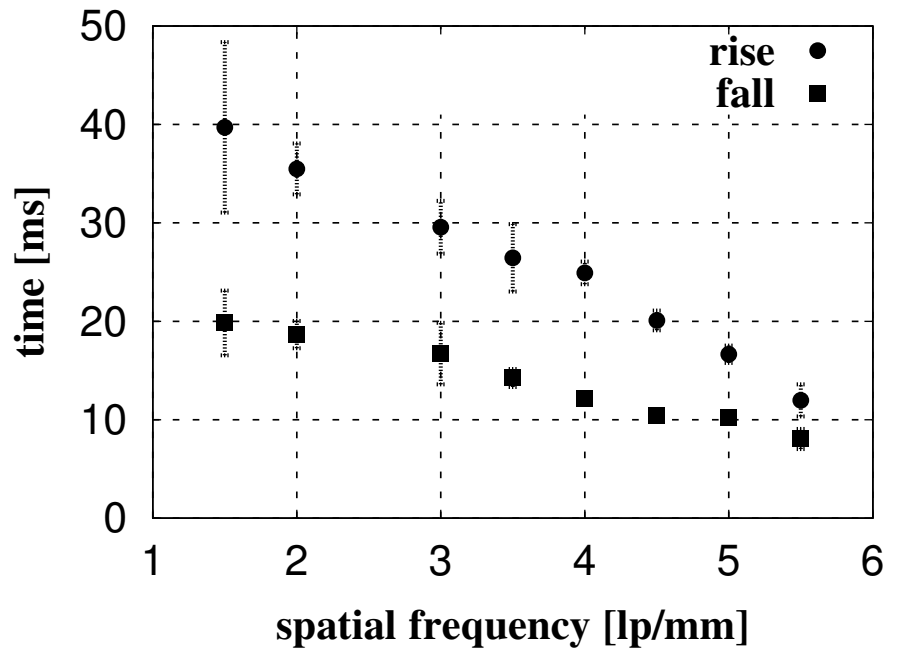

Fig. 5. The time resolution of the nitroanisole as a function of the spatial frequency of the interference fringe made by a $\mathrm{CO}_{2}$ laser. 


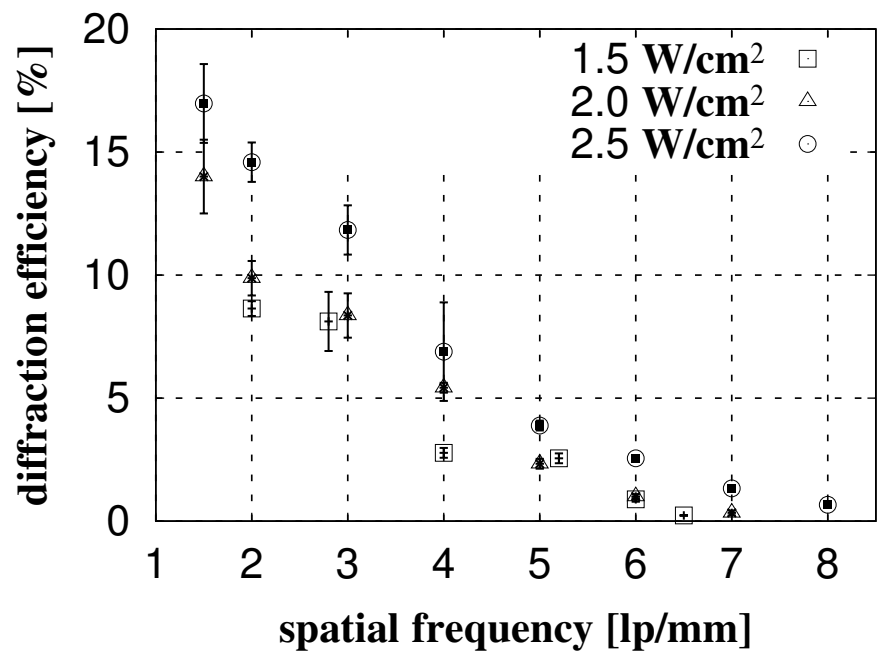

Fig. 6. Diffraction efficiency of the nitroanisole. 


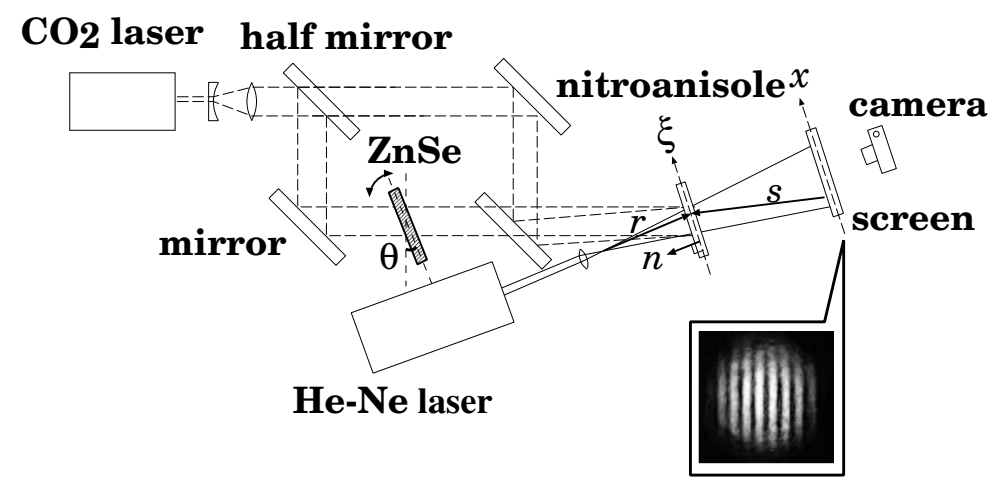

Fig. 7. Experimental setup for diffraction pattern and phase measurement. 


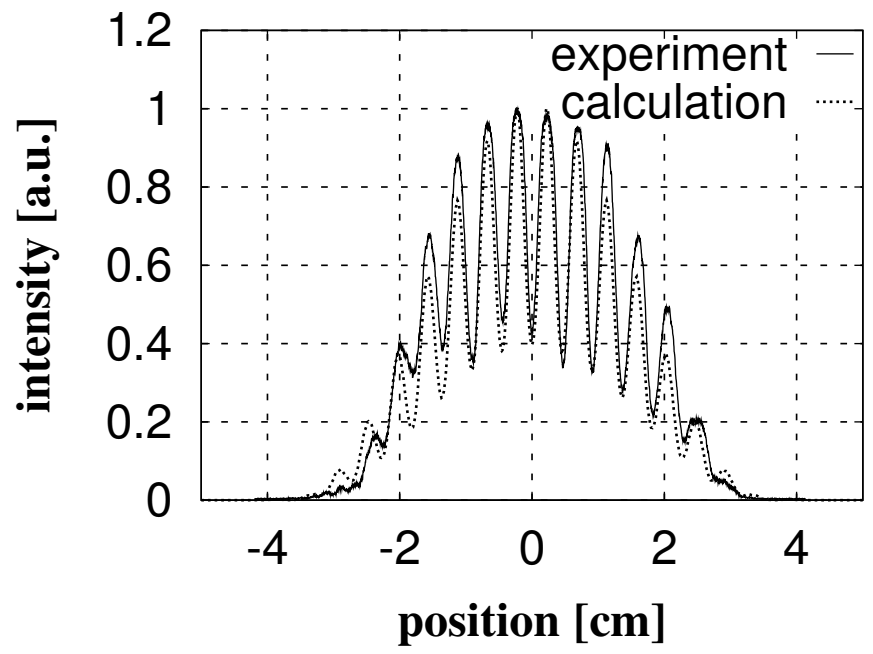

Fig. 8. Experimental results and estimation of the intensity distribution of the diffraction pattern. 


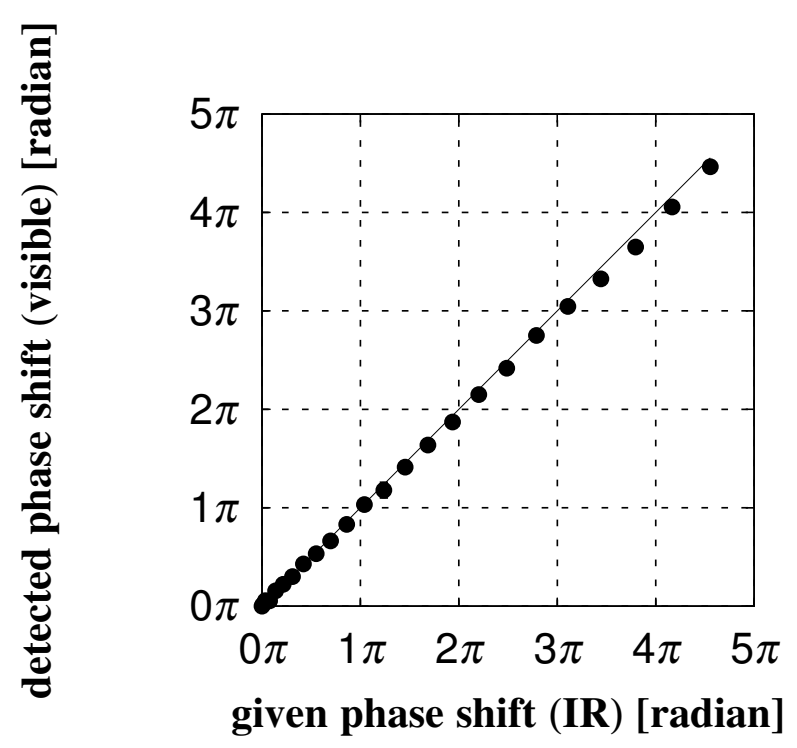

Fig. 9. The relationship between the given phase shift in mid-IR region and the detected phase shift of the visible diffraction pattern. The solid line denotes a straight line of a inclination of 1 . 


\section{References}

[1] Y. Kawano, S. Chiba, H. Shirai, A. Inoue, and A. Nagashima, Rev. Sci. Instrum. 70 (1999) 1430-1434

[2] K. Tanaka, L. N. Vyacheslavov, K. Kawahata, T. Tokuzawa, and S. Okajima, Rev. Sci. Instrum. 72 (2001) 1089-1093

[3] S. Brugioni, R. Meucchi, Opt. Commun. 230 (2004) 19-22

[4] K. Chrzanowski, S. N. Park, Infrared Phys. Techn. 43 (2001) 101-105

[5] C. M. Black, R. P. Clark, K. Darton, M. R. Goff, T. D. Norman, and H. A. Spikes, J. Biomed. Eng. 12 (1990) 281-286

[6] E. Allaria, S. Brugioni, S.D. Nicola, P. Ferraro, S. Grilli, R. Meucchi, Opt. Commun. 215 (2003) 257-262

[7] J. P. Gordon, R. C. C. Leite, R. S. Moore, S. P. S. Porte, and J. R. Whinnery, J. Appl. Phys. 36 (1965) 3-8

[8] S. J. Sheldon, L. V. Knight, and J. M. Thorne, Appl. Opt. 21 (1982) 1663-1669

[9] F. Jürgensen and W. Schröer, Appl. Opt. 34 (1995) 41-50 